\title{
A NOTE ON THE CROSS-NORM OF THE DIRECT PRODUCT OF OPERATOR ALGEBRA
}

\author{
By MaSAMichi TAKesaki
}

In [2], T. Turumaru introduced the idea of the direct product of operator algebras in the following sense. Let $\boldsymbol{M}_{1}$ and $\boldsymbol{M}_{2}$ be $C^{*}$-algebras and $\boldsymbol{M}_{1} \odot \boldsymbol{M}_{2}$ their algebraic direct product. Then we define a cross-norm $\alpha$ on $\boldsymbol{M}_{1} \odot \boldsymbol{M}_{2}$ as follows:

$\alpha\left(\sum_{i=1}^{n} x_{i} \otimes y_{i}\right)=\sup \left\{\frac{<\left(\sum_{j=1}^{m} a_{j}^{*} \otimes b_{j}^{*}\right)\left(\sum_{i=1}^{n} x_{\imath}^{*} \otimes y_{i}^{*}\right)\left(\sum_{i=1}^{n} x_{i} \otimes y_{i}\right)\left(\sum_{j=1}^{m} a_{j} \otimes b_{\jmath}\right), \varphi \otimes \psi>}{<\left(\sum_{j=1}^{m} a_{j}^{*} \otimes b_{j}^{*}\right)\left(\sum_{j=1}^{m} a_{j} \otimes b_{\jmath}\right), \varphi \otimes \psi>}\right\}^{1 / 2}$

for any element $\sum_{i=1}^{n} x_{i} \otimes y_{\imath} \in \boldsymbol{M}_{1} \odot \boldsymbol{M}_{2}$, where $\sum_{\jmath=1}^{m} a_{j} \otimes b_{\jmath}$ runs over the all non-zero elments of $\boldsymbol{M}_{1} \odot \boldsymbol{M}_{2}$ and $\varphi, \psi$ the all non-zero positive linear functionals on $\boldsymbol{M}_{1}$ and $\boldsymbol{M}_{2}$ respectively. $\boldsymbol{M}_{1} \odot \boldsymbol{M}_{2}$ becomes an imcomplete $\boldsymbol{C}^{*-}$ algebra under this cross-norm $\alpha$. We call the completion of $\boldsymbol{M}_{1} \odot \boldsymbol{M}_{2}$ the $C^{*}$-direct product of $\boldsymbol{M}_{1}$ and $\boldsymbol{M}_{2}$ and denote by $\boldsymbol{M}_{1} \widehat{\otimes}_{\alpha} \boldsymbol{M}_{2}$.

The purpose of this note is to study the relation between the $\alpha$-norm defined above and $\lambda$-norm or $\gamma$-norm in the sense of $R$. Schatten [1].

Let $\boldsymbol{M}$ be a $C^{*}$-algebra with a unit and $\subseteq$ the state space of $\boldsymbol{M}$, then $\subseteq$ is the $\sigma\left(\boldsymbol{M}^{*}, \boldsymbol{M}\right)$-convex closure of the space of all pure states on $\boldsymbol{M}$. So we call the $\sigma\left(\boldsymbol{M}^{*}, \boldsymbol{M}\right)$-closure of the space of all pure states on $\boldsymbol{M}$ the pure state space of $\boldsymbol{M}$.

Proposition 1. Let $\boldsymbol{M}_{1}$ and $\boldsymbol{M}_{2}$ be $C^{*}$-algebras with units, $\boldsymbol{M}$ their $C^{*-}$ direct product and $\Omega_{1}, \Omega_{2}$, and $\Omega$ the pure state spaces of $\boldsymbol{M}_{1}, \boldsymbol{M}_{2}$ and $\boldsymbol{M}$ respectively. Then we have

$$
\Omega=\Omega_{1} \times \Omega_{2}
$$

if and only if either $M_{1}$ or $M_{2}$ is commutative, where $\Omega_{1} \times \Omega_{2}$ means the cartesian product of $\Omega_{1}$ and $\Omega_{2}$.

Proof. Sufficiency. If $\boldsymbol{M}_{1}$ is commutative, then $\boldsymbol{M}_{1} \otimes 1$ is contained in the center $\boldsymbol{Z}$ of $\boldsymbol{M}$. Let $\tau$ be a pure state on $\boldsymbol{M}$, then we have $\langle a x, \tau\rangle$ $=\langle a, \tau\rangle\langle x, \tau\rangle$ for all $a \in Z$ and $x \in M$. If we define a linear functional $\sigma$ on $M_{1}$ such as

Received September 18, 1958. 


$$
\langle x, \sigma\rangle=\langle x \otimes 1, \tau\rangle \quad \text { for } \quad x \in M_{1},
$$

then $\sigma$ is a pure state on $\boldsymbol{M}_{1}$ by the multiplicativity of $\tau$ on $\boldsymbol{Z}$. Next we define a linear functional $\rho$ on $\boldsymbol{M}_{2}$ such as

$$
\langle y, \rho\rangle=\langle 1 \otimes y, \rho\rangle \quad \text { for } \quad y \in M_{2},
$$

then $\rho$ is a state on $M_{2}$ and $\tau=\sigma \otimes \rho$. Suppose there exist two states $\rho^{\prime}$, $\rho^{\prime \prime}$ on $M_{2}$ such as $\rho=(1 / 2)\left(\rho^{\prime}+\rho^{\prime \prime}\right)$. Put $\tau^{\prime}=\sigma \otimes \rho^{\prime}$ and $\tau=\sigma \otimes \rho^{\prime \prime}$, then $\tau=(1 / 2)\left(\tau^{\prime}+\tau^{\prime \prime}\right)$. Since $\tau$ is a pure state on $\boldsymbol{M}$, we get $\tau=\tau^{\prime}=\tau^{\prime \prime}$. We have

$$
\left\langle y, \rho>=\left\langle 1 \otimes y, \tau>=<1 \otimes y, \tau^{\prime}>=<y, \rho^{\prime}\right\rangle=\left\langle y, \rho^{\prime \prime}\right\rangle,\right.
$$

i.e. $\rho$ is a pure state on $\boldsymbol{M}_{2}$.

On the other hand, for any pure states $\sigma$ on $\boldsymbol{M}_{1}$ and $\rho$ on $\boldsymbol{M}_{2}, \tau=\sigma \otimes \rho$ is clearly a pure state on $\boldsymbol{M}$.

Combinning these arguments, the dense part of $\Omega_{1} \times \Omega_{2}$ coincides with the dense part of $\Omega$. Moreover, the mapping $(f, g) \rightarrow f \otimes g$ from $\boldsymbol{M}_{1}^{*} \times \boldsymbol{M}_{3}^{*}$ into $\boldsymbol{M}^{*}$ is weakly continuous, where the space $\boldsymbol{M}_{1}^{*} \times \boldsymbol{M}_{2}^{*}$ has the cartesian product topology of $\sigma\left(\boldsymbol{M}_{1}^{*}, \boldsymbol{M}_{1}\right)$ and $\sigma\left(\boldsymbol{M}_{2}^{*}, \boldsymbol{M}_{2}\right)$-topologies. Hence we get $\Omega=\Omega_{1} \times \Omega_{2}$.

Necessity. If $\boldsymbol{M}_{1}$ and $\boldsymbol{M}_{2}$ are both non-commutative, then there exist pure states $\sigma$ and $\rho$ on $\boldsymbol{M}_{1}$ and $\boldsymbol{M}_{2}$ respectively such that the Hilbert spaces $H_{\sigma}$ and $H_{\rho}$ canonically constructed by $\sigma$ and $\rho$ respectively are at least twodimensional. Put $\tau=\sigma \otimes \rho$, then we have $H_{\tau}=H_{\sigma} \otimes H_{\rho}$, where $H_{\tau}$ is the Hilbert space canonically constructed by $\tau$. For canonical representation $\pi_{\sigma}$, $\pi_{\rho}$ and $\pi_{\tau}$ by $\sigma, \rho$ and $\tau$, put $\pi_{\sigma}\left(\boldsymbol{M}_{1}\right)=\boldsymbol{M}_{1 \sigma}, \pi_{\rho}\left(\boldsymbol{M}_{2}\right)=\boldsymbol{M}_{2 \rho}$ and $\pi_{\tau}(\boldsymbol{M})=\boldsymbol{M}_{\tau}$ then we get $\boldsymbol{M}_{\tau}=\boldsymbol{M}_{1 \sigma} \widehat{\otimes}_{\alpha} \boldsymbol{M}_{2 \rho}$. Now for an arbitrary vector $\zeta \in H_{\tau}$ with $\|\zeta\|=1$, we define a state $\omega_{\zeta}$ on $\boldsymbol{M}_{\tau}$ such as $\left\langle z, \omega_{\zeta}\right\rangle=(z \zeta, \zeta)$ for $z \in \boldsymbol{M}_{\tau}$. Then ${ }^{t} \pi_{\tau}\left(\omega_{\zeta}\right)$ is a pure state on $\boldsymbol{M}$. If $\zeta$ is not representable by the form $\xi \otimes \eta$ for any $\xi \in H_{\sigma}$ and $\eta \in H_{\rho}$, then $\omega_{\zeta}$ is not multiplicative with respect to $\boldsymbol{M}_{1 \sigma}$ and $\boldsymbol{M}_{2 \rho}$. Hence we get ${ }^{t} \pi_{2}\left(\omega_{\zeta}\right) \notin \Omega_{1} \times \Omega_{2}$. And surely there exists such vector $\zeta$ of $H_{\tau}$ by the hypothesis on $H_{\sigma}$ and $H_{\rho}$. We get $\Omega \nsubseteq \Omega_{1} \times \Omega_{2}$. This concludes the proof.

Applying this proposition we can prove the following proposition about the cross norm of $C^{*}$-direct product of $C^{*}$-algebras.

Proposition 2. Let $\boldsymbol{M}_{1}$ and $\boldsymbol{M}_{2}$ be two $C^{*}$-algebras with units respectively. Then the $C^{*}$-norm $\alpha$ of their $C^{*}$-direct product $\boldsymbol{M}=\boldsymbol{M}_{1} \widehat{\otimes}_{\alpha} \boldsymbol{M}_{2}$ coincides with $\lambda$-norm if and only if either $\boldsymbol{M}_{1}$ or $\boldsymbol{M}_{2}$ is commutative.

Proof. Sufficiency. We assume the commutativity of $\boldsymbol{M}_{1}$. Let $\Omega_{1}$ be the pure state space of $\boldsymbol{M}_{1}$, then we have $\boldsymbol{M}_{1}=C\left(\Omega_{1}\right)$ by the well-known representation theorem of commutative $C^{*}$-algebra. For an arbitrary element $\sum_{i=1}^{n} x_{i} \otimes y_{i} \in \boldsymbol{M}_{1} \odot \boldsymbol{M}_{2}$ the $\boldsymbol{M}_{2}$-valued function $\sum_{i=1}^{n} x_{i}(t) y_{i}$ is continuous on $\Omega_{1}$, i.e. $\sum_{i=1}^{n} x_{1}(\cdot) y_{i} \in C_{\boldsymbol{M}_{2}}\left(\Omega_{1}\right)$, where $C_{\boldsymbol{M}_{2}}\left(\Omega_{1}\right)$ means the space of all $\boldsymbol{M}_{2}$-valued continuous functions with the uniform norm on $\Omega_{1}$. And we get 


$$
\begin{aligned}
\left\|\sum_{i=1}^{n} x_{i}(\cdot) y_{i}\right\| & =\sup \left\{|| \sum_{i=1}^{n} x_{i}(t) y_{i} ; t \in \Omega_{1}\right\} \\
& =\sup \left\{\mid<\sum_{i=1}^{n} x_{i}(t) y_{i}, \psi>; t \in \Omega_{1}, \psi \in \boldsymbol{M}_{2}^{*},\|\psi\| \leqq 1\right\} \\
& =\sup \left\{\mid \sum_{i=1}^{n} x_{i}(t)<y_{\imath}, \psi>; t \in \Omega_{1}, \psi \in \boldsymbol{M}_{2}^{*},\|\psi\| \leqq 1\right\} \\
& =\sup \left\{\left\|\sum_{i=1}^{n}<y_{i}, \psi>x_{i}(\cdot) ; \psi \in \boldsymbol{M}_{2}^{*},\right\| \psi \| \leqq 1\right\} \\
& =\sup \left\{\mid \sum_{i=1}^{n}<x_{i}, \varphi><y_{i}, \psi>; \psi \in \boldsymbol{M}_{1}^{*},\|\varphi\| \leqq 1, \psi \in \boldsymbol{M}_{2}^{*},\|\psi\| \leqq 1\right\} \\
& =\lambda\left(\sum_{i=1}^{n} x_{i} \otimes y_{i}\right) .
\end{aligned}
$$

Moreover, if we define a multiplication and a *-operation of $C_{\boldsymbol{M}_{2}}\left(\Omega_{1}\right)$ as follows:

$$
(a \cdot b)(t)=a(t) b(t), \quad(a)^{*}(t)=a(t)^{*} \quad \text { for all } a, b \in C_{M_{2}}\left(\Omega_{1}\right)
$$

and $t \in \Omega_{1}$, then $C_{M_{2}}\left(\Omega_{1}\right)$ becomes a $C^{*}$-algebra since we have

$$
\begin{aligned}
\left\|a^{*} a\right\| & =\sup \left\{\left\|\left(a^{*} a\right)(t)\right\| ; t \in \Omega_{1}\right\} \\
& =\sup \left\{\left\|a^{*}(t) a(t)\right\| ; t \in \Omega_{1}\right\} \\
& =\sup \left\{\|a(t)\|^{2} ; t \in \Omega_{1}\right\} \\
& =\sup \left\{\|a(t)\| ; t \in \Omega_{1}\right\}^{2} \\
& =\|a\|^{2}
\end{aligned}
$$

for all $a \in C_{\mathbf{M}_{2}}\left(\Omega_{1}\right)$.

Considering the natural correspondence of $\sum_{i=1}^{n} x_{i} \otimes y_{\imath} \in M_{1} \widehat{\otimes}_{\lambda} M_{2}$ and $\sum_{i=1}^{n} x_{i}(\cdot) y_{i} \in C_{\boldsymbol{M}_{2}}\left(\Omega_{1}\right), \quad \boldsymbol{M}_{1} \widehat{\otimes}_{\lambda} \boldsymbol{M}_{2}$ becomes a $C^{*}$-algebra and the totality of linear functionals of the form $\varphi \otimes \psi$, where $\varphi$ and $\psi$ are positive linear functionals on $\boldsymbol{M}_{1}$ and $\boldsymbol{M}_{2}$ respectively, is total on $\boldsymbol{M}_{1} \widehat{\otimes}_{\lambda} \boldsymbol{M}_{2}$. Hence we get

$$
\begin{aligned}
\lambda\left(\sum_{i=1}^{n} x_{i} \otimes y_{i}\right) & =\sup \left\{\begin{array}{c}
<\left(\sum_{j=1}^{m} a_{\jmath}^{*} \otimes b_{j}^{*}\right)\left(\sum_{i=1}^{n} x_{i}^{*} \otimes y_{i}^{*}\right)\left(\sum_{i=1}^{n} x_{i} \otimes y_{i}\right)\left(\sum_{j=1}^{m} a_{j} \otimes b_{\jmath}\right), \psi \otimes \psi> \\
<\left(\sum_{j=1}^{m} a_{j}^{*} \otimes b_{j}^{*}\right)\left(\sum_{j=1}^{m} a_{j} \otimes b_{\jmath}\right), \varphi \otimes \psi>
\end{array}\right\}^{1 / 2} \\
& =\alpha\left(\sum_{i=1}^{n} x_{i} \otimes y_{i}\right)
\end{aligned}
$$

for all $\sum_{i=1}^{n} x_{i} \otimes y_{i} \in \boldsymbol{M}_{1} \odot \boldsymbol{M}_{2}$, where $\sum_{\jmath=1}^{m} a_{j} \otimes b_{\jmath}$ runs over the all non-zero elements of $\boldsymbol{M}_{1} \odot \boldsymbol{M}_{2}$ and $\psi, \psi$ the all non-zero positive linear functionals on $\boldsymbol{M}_{1}$ and $\boldsymbol{M}_{2}$ respectively. Therefore we get $\alpha=\lambda$.

Indeed, we have $\boldsymbol{M}_{1} \widehat{\otimes}_{\lambda} \boldsymbol{M}_{2}=\boldsymbol{C}_{\boldsymbol{M}_{2}}\left(\Omega_{1}\right)$ by the decomposition of identity.

Necessity. We assume $\alpha=\lambda$. Then we have

$$
\alpha(u)=\sup \left\{|<u, f>| ; f \in \Sigma_{1} \otimes \Sigma_{2}\right\} \quad \text { for all } u \in \boldsymbol{M},
$$


where $\Sigma_{1}$ and $\Sigma_{2}$ are unit spheres of $\boldsymbol{M}_{1}^{*}$ and $\boldsymbol{M}_{2}^{*}$ respectively, and $\Sigma_{1} \otimes \Sigma_{2}$ means the totality of $\varphi \otimes \psi\left(\varphi \in \Sigma_{1}, \psi \in \Sigma_{2}\right)$. Hence if $\Sigma$ is the unit sphere of $\boldsymbol{M}^{*}$, we get

$$
\left.\Sigma_{1} \otimes \Sigma_{2}\right)^{\circ 0}=\Sigma
$$

where $\left(\Sigma_{1} \otimes \Sigma_{2}\right)^{\circ \circ}$ means the bipolar of $\Sigma_{1} \otimes \Sigma_{2}$. $\quad \sum_{1} \otimes \sum_{2}$ is $\sigma\left(\boldsymbol{M}^{*}, \boldsymbol{M}\right)$ compact so that all extreme points of $\Sigma$ belong to $\Sigma_{1} \otimes \Sigma_{2}$ by Krein-Milman's Theorem. Hence we have $\Omega \subset \Sigma_{1} \otimes \Sigma_{2}$, where $\Omega$ is the pure state space on $\boldsymbol{M}$. So the pure state on $\boldsymbol{M}$ is multiplicative with respect to $\boldsymbol{M}_{1}$ and $\boldsymbol{M}_{2}$. For a pure state $\tau$ on $\boldsymbol{M}$, put

$$
\begin{array}{ll}
\langle x, \sigma>=\langle x \otimes 1, \tau> & \text { for } \quad x \in M_{1}, \\
<y, \rho>=\langle 1 \otimes y, \rho> & \text { for } \quad y \in M_{2},
\end{array}
$$

then $\sigma$ and $\rho$ are pure states on $\boldsymbol{M}_{1}$ and $\boldsymbol{M}_{2}$ respectively and $\tau=\sigma \otimes \rho$. That is, we get

$$
\Omega=\Omega_{1} \times \Omega_{2},
$$

where $\Omega_{2}$ is the pure state space $\boldsymbol{M}_{2}$. Therefore either $\boldsymbol{M}_{1}$ or $\boldsymbol{M}_{2}$ is commutative by Proposition 1. This concludes the proof.

\section{REFERENCES}

[1] Schatten, R., Theory of cross-spaces. Princeton, (1950).

[2] Turumaru, T., On the direct product of operator algebras I. Tôhoku Math. Journ. 4 (1954), 242-251.

DEPARTMENT OF MATHEMATICS,

Tokyo Institute of Technology. 